\title{
Magnetic field turbulence, electron heating, magnetic holes, proton cyclotron waves, and the onsets of bipolar pulse (electron hole) events: a possible unifying scenario
}

\author{
B. T. Tsurutani ${ }^{1}$, B. Dasgupta ${ }^{1}$, J. K. Arballo ${ }^{1}$, G. S. Lakhina ${ }^{2}$, and J. S. Pickett ${ }^{3}$ \\ ${ }^{1}$ Jet Propulsion Laboratory, California Institute of Technology, Pasadena, CA, USA \\ ${ }^{2}$ Indian Institute of Geomagnetism, Mumbai/Bombay 400 005, India \\ ${ }^{3}$ Department of Physics and Astronomy, University of Iowa, Iowa City, IA, USA
}

Received: 31 December 2001 - Revised: 19 August 2002 - Accepted: 12 September 2002

\begin{abstract}
Two electron heating events have been identified on 20 May 1996 when Polar was in the polar cap/polar cusp boundary layer. The electron heating events were located within magnetic holes/cavities/bubbles and were accompanied by nonlinear $\pm 14 \mathrm{nT}$ peak-to-peak ( $\mathrm{f} \sim 0.6$ to $0.7 \mathrm{f}_{\mathrm{cp}}$ ) obliquely propagating proton cyclotron waves. The electrons appear to be heated isotropically. Electric bipolar pulse (electron hole) onset events were also detected within the heating events. We propose a scenario which can link the above phenomena. Nonlinear Alfvén waves, generated through cusp magnetic reconnection, propagate down magnetic field lines and locally heat electrons through the ponderomotive force. The magnetic cavity is created through the diamagnetic effect of the heated electrons. Ion heating also occurs through ponderomotive acceleration (but much less than the electrons) and the protons generate the electromagnetic proton cyclotron waves through the loss cone instability. The obliquely propagating electromagnetic proton cyclotron waves accelerate bi-streaming electrons, which are the source of free energy for the electron holes.
\end{abstract}

\section{Introduction}

Magnetic holes (Cummings and Coleman, 1968; Sugiura et al., 1969) are regions of local decrease in magnetospheric magnetic field magnitudes that contain enhanced plasma densities maintaining pressure balance (Lühr and Klöcher, 1987; Treumann et al., 1990; Stasiewicz et al., 2001). This auroral zone magnetic field phenomena have also been called magnetic cavities and magnetic bubbles. Low frequency ( $\mathrm{f}<25 \mathrm{~Hz}$ ) electromagnetic turbulence often accompany the magnetic holes. Treumann et al. (1990) have indicated that

Correspondence to: B. T. Tsurutani

(bruce.t.tsurutani@jpl.nasa.gov) the holes contain electromagnetic waves with frequencies up to several times the ion cyclotron frequency which may be either whistler waves or ion cyclotron waves. Stasiewicz et al. (2001) have analyzed waves in the $0-30 \mathrm{~Hz}$ frequency range and have argued that these waves are Doppler-shifted kinetic Alfvén waves (KAWs).

Stasiewicz et al. (2001) have suggested that the magnetic holes are created by a tearing mode reconnection process and that the related $0.1-30 \mathrm{~Hz}$ broadband waves are most likely Alfvén waves generated by the Hall instability. The plasma heating is believed to be related to particle interactions with kinetic Alfvén waves.

Pickett et al. (2002) have analyzed the wave turbulence in the outer cusp boundary layer to attempt to identify the nature of the turbulence. They have found that the turbulence E/B ratios did not exactly fit the kinetic Alfvén wave (KAW) dispersion curve (contrary to Stasiewicz et al., 2001) and Pickett et al. concluded that the waves might be a mixture of dispersive KAWs and perhaps lower hybrid turbulence.

Angelopoulos et al. (2001) have found that the low frequency wave power is consistent with 2-D turbulence with phase velocities smaller than the spacecraft speed and frequency as low as or lower than the oxygen gyrofrequency. They conclude that these waves cannot resonate with and heat the ions.

The purpose of this paper is to examine two magnetic hole events in detail using a full complement of Polar field and plasma data. At the time of the events, the Polar spacecraft was at an altitude close to apogee $\left(\sim 6 R_{E}\right)$ and within the polar cap/cusp boundary layer. Wideband plasma wave, highresolution electron and ion data and high resolution magnetic field data are used in the analyses. In conclusion, we will present a possible scenario that links Alfvén waves, electron heating, magnetic holes, proton cyclotron waves and onsets of electric bipolar pulse (electron hole) events. 


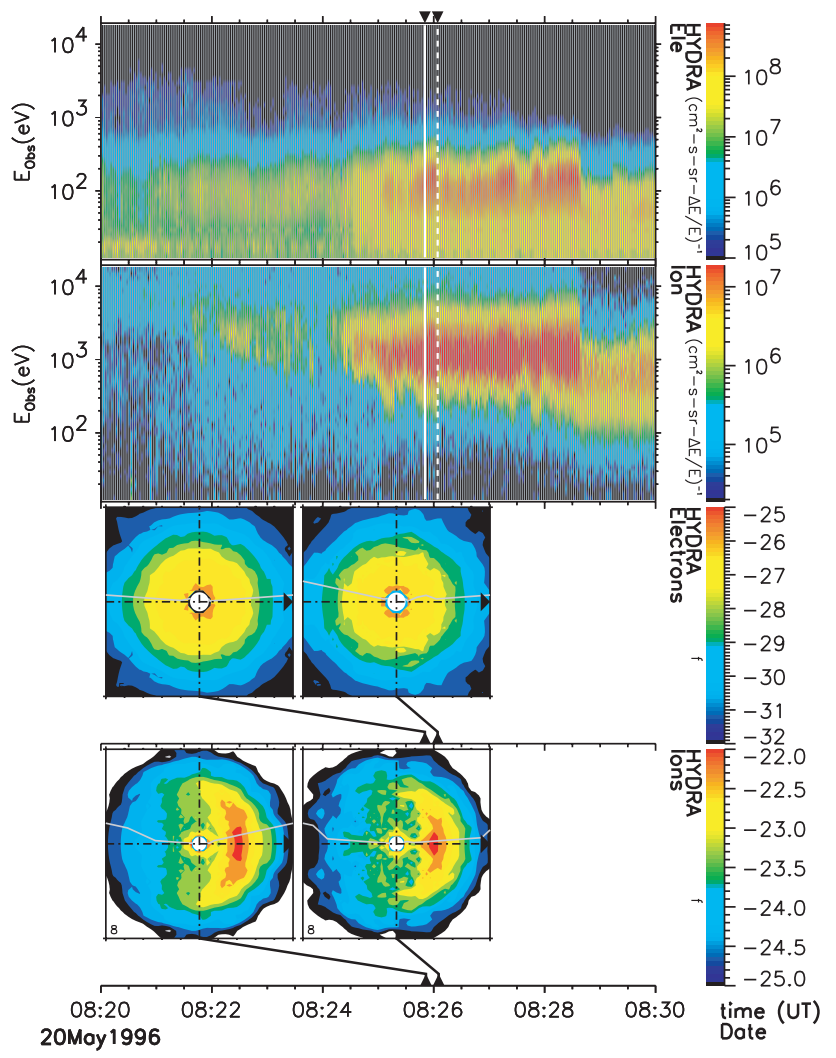

Fig. 1. An electron heating event. The vertical solid white line in the top two panels marks the time of the electron heating event. The vertical dashed line is a time of a "background" interval. The third and fourth rows show the electron and ion 2-D distributions, respectively. The distributions on the left are at the time of the electron heating event. Those on the right are during the "background" interval. The electrons are heated isotropically.

\section{Data analyses}

We use the Polar satellite plasma wave, plasma and magnetometer high-time resolution data for the study. The instrument descriptions can be found in Gurnett et al. (1995), Scudder et al. (1995) and Russell et al. (1995), respectively. The Polar spacecraft was located near local noon at $\sim 08: 26$ UT, 20 May 1996, for the events of concern.

\section{Results}

\subsection{Electron heating}

Figure 1 shows the plasma environment during the time of one of the magnetic hole events. The top panel gives the electrons and the second panel the ions. The Hydra instrument does not distinguish mass (however, since the predominant ions present in the boundary layers are protons, we will hereafter make the assumption that these are protons). The vertical scale is particle energy, the horizontal scale is time, and the particle flux is given by the color. The onset time of the first electron heating event is indicated by a vertical solid

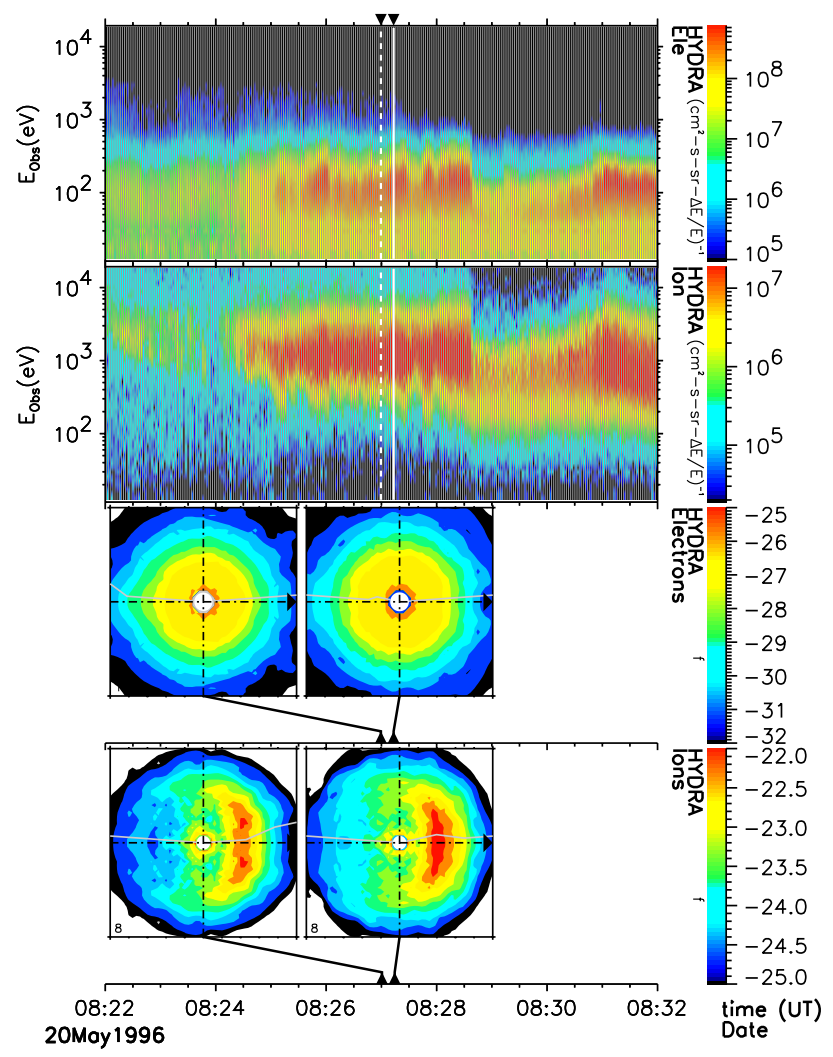

Fig. 2. A second electron heating event in the same format as in Fig. 1. The electrons are again heated isotropically.

line. A vertical dashed line gives a "background" time to be used for comparative purposes.

It can be noted that the electron heating occurs simultaneously with electron and ion density increases. This is apparent by the broadening of the energy ranges of both types of particles (both higher and lower energies are apparent at these times) and the brightening in color. The electrons are heated to $\sim 100-200 \mathrm{eV}$ (note the "background" is already relatively high. This will be discussed later).

The electron distribution functions during the "background interval" and at the time of the first electron heating event are shown in the next to bottom right and next to the bottom left distribution plots, respectively. The distribution functions are determined by using data from two spacecraft spins, or $\sim 13 \mathrm{~s}$. The proton distribution functions during the "background interval" and the electron heating event are given in the bottom right and left panels, respectively. The magnetic field direction is given by the horizontal dotdashed lines and black arrows (on the far right of each panel). The solid triangles at the very bottom give the times for the distribution functions.

Several important features can be noted in the distribution plots. The electron distributions (third panels from top) during the background interval and during the electron heating interval are isotropic. The electron temperatures are enhanced during the ion event (note the larger radius of the yel- 
low and aqua regions). This can also be seen in the top panel where electrons at $\sim 200 \mathrm{eV}$ are noted. There is no evidence of electron bi-directional streaming in either case.

There is a field-aligned ion beam during the background interval. For the electron heating interval, the particle fluxes are enhanced and distended in $V_{\perp}$ relative to the background (right side) case. Fluxes are enhanced at pitch angles $\leq$ $\pm 45^{\circ}$. Thus the "change" in the ion distribution during the event is that the field-aligned beam widens to form a "broad beam" with pitch angles throughout $\sim \pm 45^{\circ}$ pitch.

Figure 2 is in the same format as Fig. 1, but for the second electron heating event. The vertical dashed line is the background and the vertical solid line is the time of the electron event.

The distributions on the left are the background electrons and ions, and the distributions on the right are the electrons and ions for the electron heating event. The background distribution for this second event are similar to those of the first event. For the background case, the electrons are isotropic. When the electrons are heated, they are again heated isotropically. During background, the ions have a pitch angle distribution including particles of pitch within $\sim \pm 30^{\circ}$ of $\boldsymbol{B}_{0}$. During electron intensification, ion fluxes are increased and the edge of the cone of pitch angles increased to $\pm 45^{\circ}$.

\subsection{Holes/cavities/bubbles}

The three components of the magnetic field and field magnitude are shown in spacecraft coordinates in Fig. 3. In this system, $\hat{x}$ is along the projection to the sun in the spin plane, $\hat{z}$ is the rotation axis of the Polar spacecraft, and $\hat{y}$ forms a right-hand system. The two electron heating events are indicated by vertical dashed lines. The electron events are associated with intervals where there are magnetic field magnitude decreases (next to bottom panel) as well as corresponding electron density enhancements (bottom panel). These are the magnetic holes (cavities/bubbles) that have been previously reported in other studies (Lühr and Klöcher, 1987, Treumann et al., 1990; Stasiewicz et al., 2001).

The magnetic field structures are shown in high-time resolution in Fig. 4. The field components are given in a minimum variance coordinate system, where $B_{1}, B_{2}$ and $B_{3}$ are the components along the maximum, intermediate, and minimum variance directions, respectively. Dashed vertical lines include intervals where the minimum variances were calculated.

The minimum variance method has been described by Sonnerup and Cahill (1967) and first applied to electromagnetic wave analyses by Smith and Tsurutani (1976). The results of the analyses are shown in the hodograms of Fig. 5. The analyses of the first interval from 08:25:17.2 to 08:26:06.4 UT is given on the left. Assuming that this whole interval is one cycle of a wave, the wave is found to be linearly/elliptically polarized $\left(\lambda_{1} / \lambda_{2}=8.4\right)$ and is propagating at a $\sim 79^{\circ}$ angle relative to the ambient magnetic field. There are higher frequency waves superposed on this low frequency wave. The former waves will be discussed later. The sec-

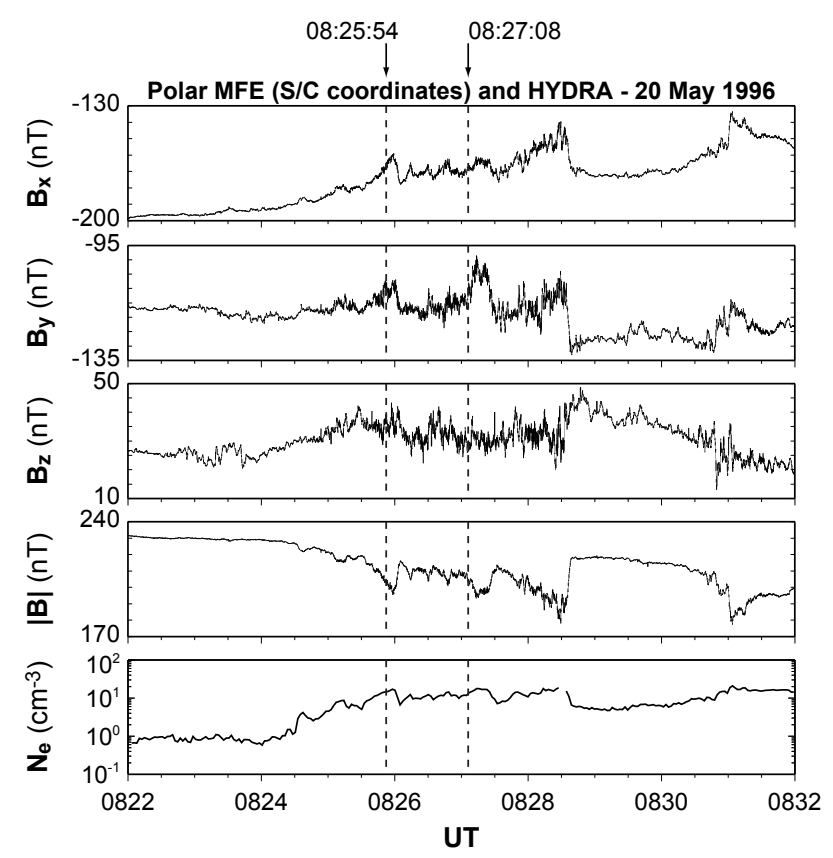

Fig. 3. The magnetic field in spacecraft coordinates (top four panels) and the electron density (bottom panel). The times of the two electron heating events are indicated by vertical dashed lines. The electron heating events occur in regions of magnetic field depressions and of ions and electron density enhancements.

ond wave event occurs between 08:26:39.8 to 08:27:28.9 UT. This wave is elliptically polarized $\left(\lambda_{1} / \lambda_{2}=3.9\right)$ and is propagating at $\sim 54^{\circ}$ relative to $\boldsymbol{B}_{0}$. In the above, $\lambda_{1}$ and $\lambda_{2}$ are the maximum and intermediate eigenvalues of the covariance matrix. The polarization is similar in both wave cases, and the oblique propagation characteristics are also similar.

The power spectra for the second electron heating event is shown in Fig. 6 (the results for the first event are similar, but is not shown for brevity). The time interval shown is from 08:26:20 to 08:27:50 UT, 20 May 1996. The coordinate system used is the minimum variance system. The spectrum on the left is the power in the direction of maximum variance for the interval. The spectrum is more or less a steep power law $\left(\mathrm{f}^{-2.3}\right)$ extending from $5 \cdot 10^{-2} \mathrm{~Hz}$ to $7 \cdot 10^{-1} \mathrm{~Hz}$. Particularly noticeable is a peak at $\sim 2 \mathrm{~Hz}$. The right panel shows the power spectra in the field magnitude. The spectral features are similar to the left-hand panel. There is a steep power law spectrum from $5 \cdot 10^{-2}$ to $7 \cdot 10^{-1} \mathrm{~Hz}$. The slope is slightly steeper $\left(\mathrm{f}^{-2.6}\right)$. There is again a distinct peak at $\sim 2 \mathrm{~Hz}$. It is noted that the spectral shape of interplanetary magnetic fluctuations at $1 \mathrm{AU}$ is also a power law, but less steep. Typical spectra are $\mathrm{f}^{-1.6}$ to $\mathrm{f}^{-1.8}$ (see Table 2 in Tsurutani et al., 2002a). The significance of this comparison will be discussed later.

\subsection{Electromagnetic $\sim 2 \mathrm{~Hz}$ waves}

To get more information concerning the nature of the spectral peaks near $\sim 2 \mathrm{~Hz}$, we analyze the output of the PWI low- 


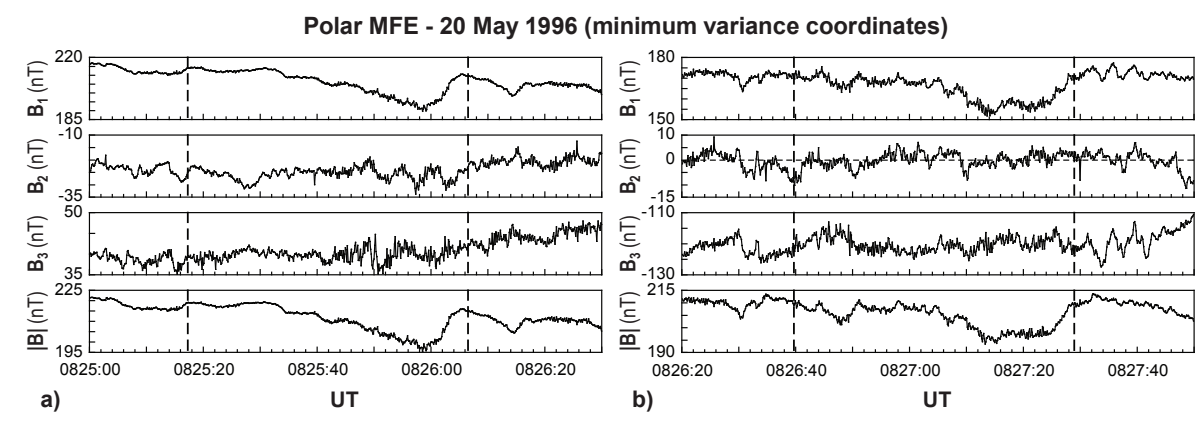

Fig. 4. The two panels correspond to the magnetic fields during and around two electron heating intervals. In each panel the interval between the two vertical dashed lines were used to define the minimum variance coordinate system, the system used to display the field components.

Polar MFE - 20 May 1996 (minimum variance coordinates)

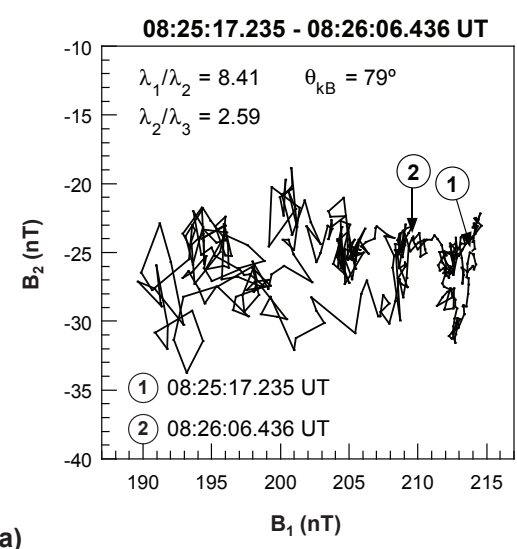

a)

Fig. 5. The hodogram for the two intervals of Fig. 4. The authors suggest that these intervals are single cycles of Alfvén waves. The waves are linearly/elliptically polarized and are propagating at $79^{\circ}$ and $54^{\circ}$ relative to the ambient magnetic field, respectively.

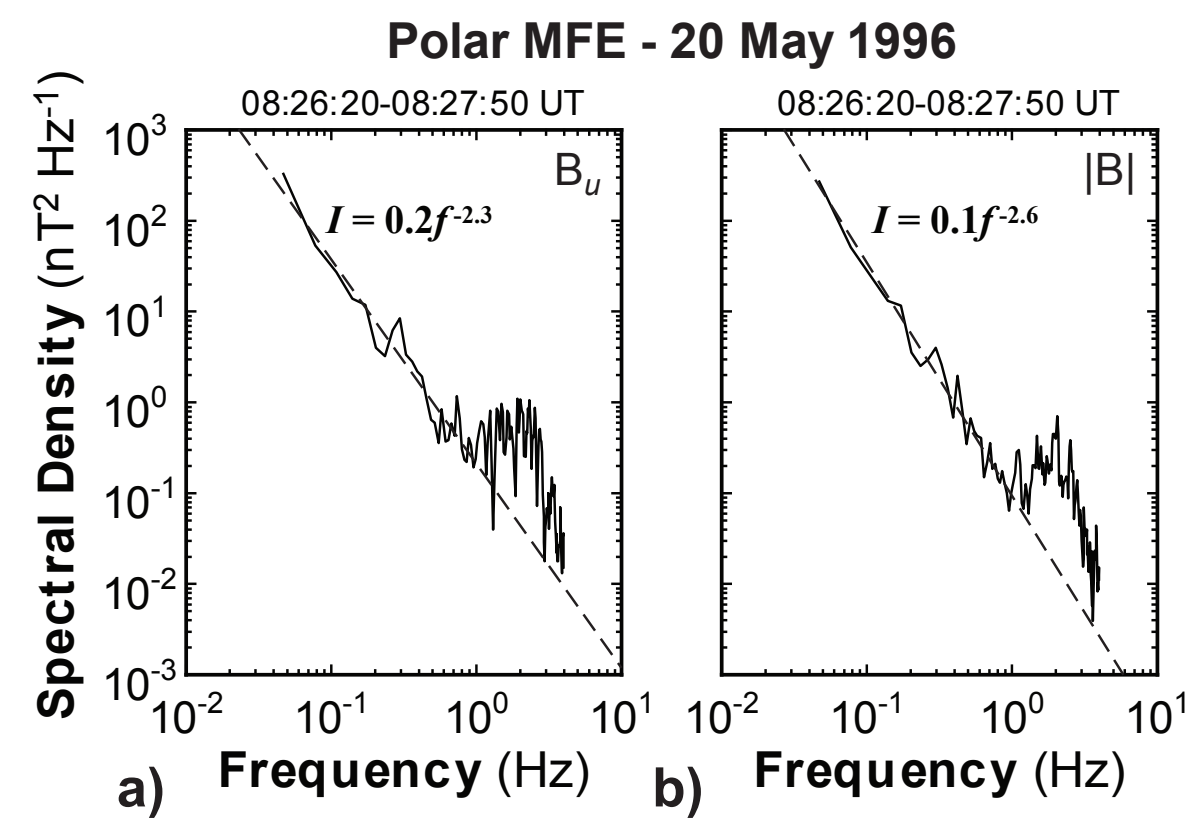

Fig. 6. Power spectra of the magnetic field for the interval 08:26:20 to 08:27:50 UT. The left panel is for the component along the maximum variance direction. The right panel is for the field magnitude. Both spectra display power law forms with a superposed peak at $\sim 2 \mathrm{~Hz}$. 


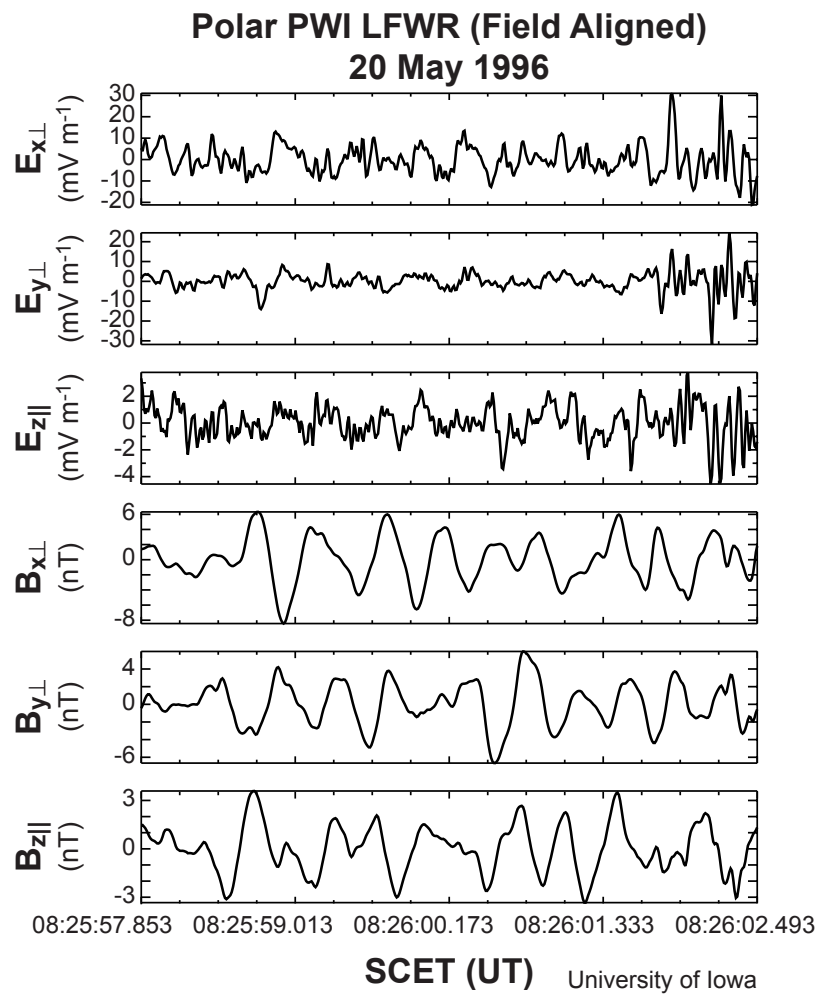

$$
\begin{array}{rlrl}
\mathrm{R}_{\mathrm{E}} & =6.05 & & \\
\mathrm{~L} & =27.32 & \mathrm{MLT} & =11.62 \\
\lambda_{\mathrm{m}} & =61.96 & \mathrm{~F}_{\mathrm{cp}} & =3.05 \mathrm{~Hz}
\end{array}
$$

Fig. 7. Low frequency $(\mathrm{f}>1 \mathrm{~Hz}$ ) waves during the first electron heating onset. The bandpass is $1 \mathrm{~Hz}$ to $25 \mathrm{~Hz}$. The coordinates are magnetic field-aligned. Electromagnetic waves with peak-topeak amplitudes as large as $\sim 14 \mathrm{nT}$ are detected. The waves are mixed left-hand and right-hand polarized in the spacecraft frame. The $\sim 2.3 \mathrm{~Hz}$ waves occur at $\sim 0.75 \mathrm{f}_{\mathrm{cp}}$ and are thus believed to be proton cyclotron waves.

frequency wave form receiver (LFWR) above $\sim 1 \mathrm{~Hz}$ for the above two events. The results are shown in Figs. 7 and 8. Figure 7 corresponds to the time interval of 08:25:57.853 to $08: 26: 02.493$ UT on 20 May 1996 and contains the time around the first electron heating event. Figure 8 shows the time interval of $08: 27: 11.452$ to $08: 27: 16.092$ UT on 20 May 1996 at approximately the time of the second electron heating event. In both figures, the data are plotted in field-aligned coordinates, where $\hat{z}$ is in the direction of the magnetic field, $\hat{y}$ is in the dawn-dusk direction orthogonal to $\hat{z}$, and $\hat{x}$ completes the right-hand coordinate system. The top three panels show the electric field components and the lower three panels the magnetic field components for fluctuations above $1 \mathrm{~Hz}$.

Figure 7 shows the clear presence of large amplitude electromagnetic waves. The transverse magnetic field component is as large as $\sim 14 \mathrm{nT}$ peak-to-peak (at $\sim 08: 25: 58.903$ UT) with a significant parallel component of $\sim 7 \mathrm{nT}$ peak-to-peak. The average wave period is $0.44 \mathrm{~s}$ or a frequency of $\sim 2.3 \mathrm{~Hz}$. The local proton cyclotron fre-

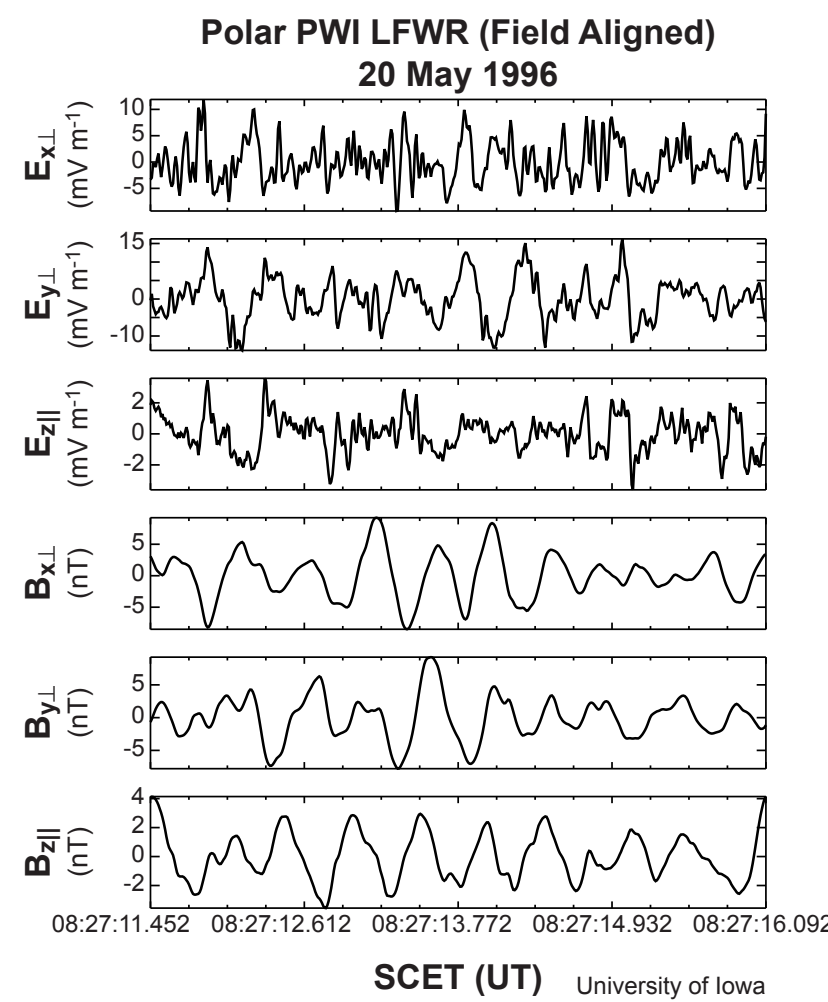

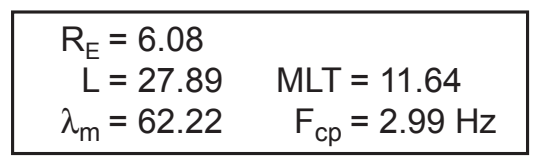

Fig. 8. Same as Fig. 6, but for the second electron heating event. The magnetic wave amplitudes are as large as $\sim 9 \mathrm{nT}$ peak-to-peak with a parallel component of $3 \mathrm{nT}$. The wave frequency is $\sim 1.9 \mathrm{~Hz}$ or $0.63 \mathrm{f}_{\mathrm{cp}}$. The waves are left-hand elliptically polarized in the spacecraft frame, propagating at an angle of $\sim 70^{\circ}$ relative to $\boldsymbol{B}_{0}$. The waves are electromagnetic proton cyclotron waves.

quency is $3.05 \mathrm{~Hz}$. Thus the wave frequency is $0.75 \mathrm{f}_{\mathrm{cp}}$. Minimum variance analyses have been applied to the waves (not shown). The waves appear to be a mixture of left-and rightpolarized waves propagating obliquely to $\boldsymbol{B}_{0}$. Detailed analyses of this event is beyond the scope of the present paper and will be postponed for subsequent analyses.

There are significant wave electric fields present as well. The most interesting are the large components parallel to $\boldsymbol{B}_{0}$. There is a $\sim 4 \mathrm{mV} \mathrm{m}^{-1}$ component that is part of the obliquely propagating electromagnetic waves. However this is not the only wave component that is present. At $\sim 08: 26: 02.333$ UT to 08:26:02.493 UT, there are high frequency $\sim 6 \mathrm{mV} \mathrm{m}^{-1}$ peak-to-peak waves present. These electrostatic waves have a frequency of $\sim 31 \mathrm{~Hz}$. We note that the electrostatic waves at $\sim 31 \mathrm{~Hz}$ are occurring at frequencies above the bandpass roll off frequency of $25 \mathrm{~Hz}$ (the $3 \mathrm{~dB}$ level). It is estimated that the attenuation at $31 \mathrm{~Hz}$ is $20 \mathrm{~dB}$. Thus, the true amplitude of the electric waves may be extremely large. The existence of wave components at frequen- 


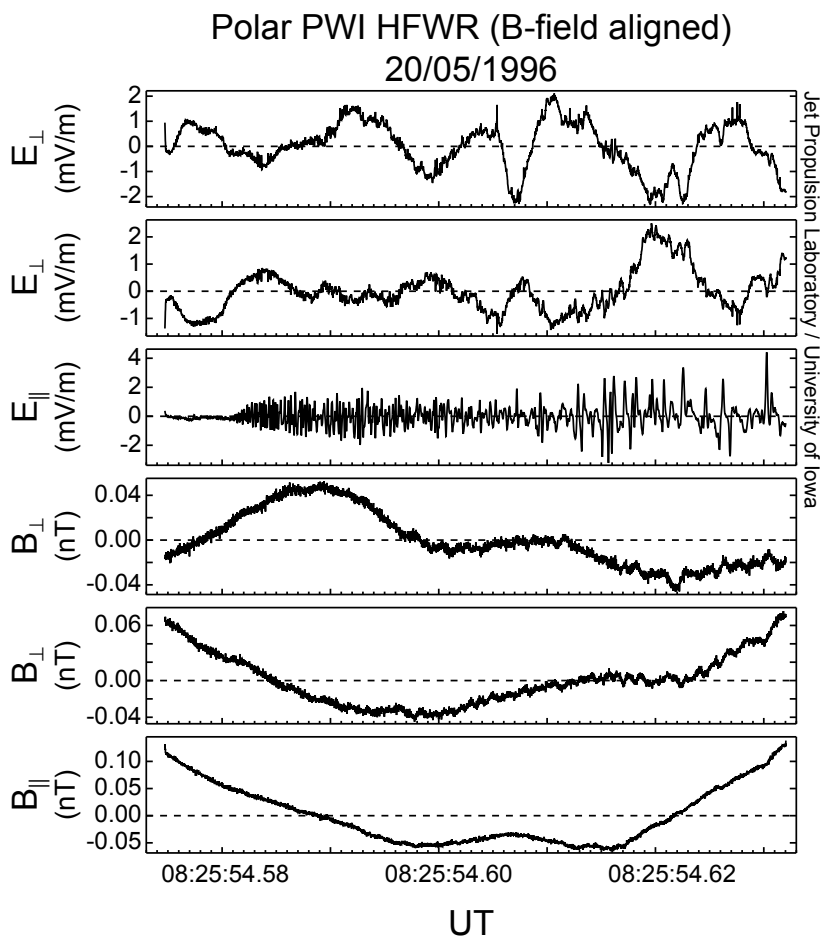

Fig. 9. A bipolar pulse (electron hole) event onset at $\sim 08: 25: 55$ UT, during the time of the first electron heating event. The bandpass is $25 \mathrm{~Hz}$ to $25 \mathrm{kHz}$. The electric signals are purely parallel to $\boldsymbol{B}_{0}$. The wave growth rate is $\sim 0.25 \mathrm{f}_{\text {ce }}$.

cies above $31 \mathrm{~Hz}$ cannot be addressed by this data set. In comparison, the lower hybrid frequency $\sqrt{\mathrm{f}_{\mathrm{ce}} \mathrm{f}_{\mathrm{cp}}}$ is $\sim 240 \mathrm{~Hz}$.

Figure 8 shows the presence of electromagnetic proton cyclotron waves during the second electron heating event. The wave amplitudes are as large as $\sim 9 \mathrm{nT}$ peak-to-peak with a parallel component of $\sim 3 \mathrm{nT}$ peak-to-peak. The average wave period is $0.53 \mathrm{~s}$ or $\mathrm{f} \sim 1.9 \mathrm{~Hz}$. This corresponds to $\mathrm{f} \cong 0.63 \mathrm{f}_{\mathrm{cp}}$. The waves are left-hand elliptically polarized and propagate at an angle $\sim 70^{\circ}$ relative to $\boldsymbol{B}_{0}$. The maximum-to-intermediate eigenvalue ratio $\lambda_{1} / \lambda_{2}$ is 19 , indicating the waves are highly elliptically polarized. There are also significant parallel electric field components with $E_{\|} \sim 2-4 \mathrm{mV} \mathrm{m}^{-1}$. Again, much of the electric oscillations are associated with the obliquely propagating proton cyclotron waves, but there is also a high frequency wave component present as well, similar to the case in Fig. 7.

\subsection{Electrostatic bipolar pulses}

An electric bipolar pulse onset event that occurred during the first electron heating event is shown in Fig. 9. The event onset occurs at $\sim 08: 25: 55$ UT. At $\sim 08: 25: 54.581$ UT the $E_{\|}$signal (third panel from the top) appears out of background. It reaches a peak-to-peak amplitude of $\sim 2 \mathrm{mV} \mathrm{m}^{-1}$ by $08: 25: 54.584 \mathrm{UT}$. The rise time duration is $\sim 3 \cdot 10^{-3} \mathrm{~s}$. There is little or no $E_{\perp}$ (topmost two panels) component in these signals. The local electron cyclotron frequency was $\sim 5.7 \cdot 10^{3} \mathrm{~Hz}$ at the time of the event. Assuming an expo-

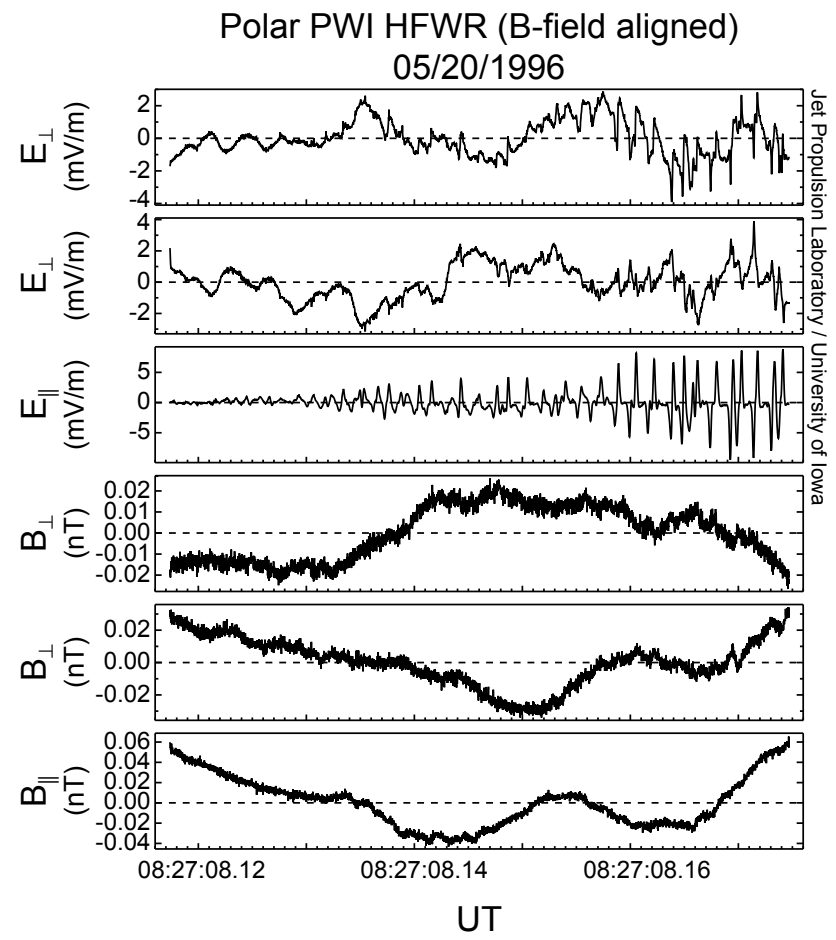

Fig. 10. Same format as Fig. 8, for a bipolar pulse event onset at $\sim$ 08:27:08 UT. Peak parallel wave amplitudes reach $\pm 9 \mathrm{mV} \mathrm{m}^{-1}$.

nential growth rate, Tsurutani et al. (2001a) had determined a wave growth rate of $\sim 0.25 \mathrm{f}_{\mathrm{ce}}$.

A bipolar pulse onset event occurred at $\sim 08: 27: 08 \mathrm{UT}$, during the second electron heating event. This is shown in Fig. 10. Again, the signals rise out of noise (at $\sim 08: 27: 08.120 \mathrm{UT}$ ) and the signals are (initially) present only in $E_{\|}$. The waves reach an amplitude of $\sim$ $\pm 2 \mathrm{mV} \mathrm{m}^{-1}$ by $08: 27: 08.137 \mathrm{UT}$. After a slight lull, the wave amplitude grew again and reached $\sim \pm 9 \mathrm{mV} \mathrm{m}^{-1}$ by $\sim 08: 27: 08.169$ UT. By this time, the transverse $\left(E_{\perp}\right)$ electric components reached amplitudes of $\sim 3-4 \mathrm{mV} \mathrm{m}^{-1}$. This wave growth rate was approximately five times slower than that of the Fig. 9 event.

\section{Summary}

We have shown the simultaneous presence of many plasma/plasma wave phenomena in a region of the polar cap/cusp boundary layer. Electron heating events and ion density enhancements are detected in magnetic holes/cavities/bubbles. The ion events have many features similar to cusp injection events (Savin et al., 1998; Pickett et al., 2002). Large amplitude, obliquely propagating electromagnetic waves with frequencies slightly below the local proton cyclotron frequency were also detected during the electron heating events. From the frequency and polarization characteristics, these can be identified as electromagnetic proton cyclotron waves (other modes are clearly present as well, but have yet to be identified). Electric bipolar pulse 
(electric hole) onsets are detected with the electron heating events. There were no long-lasting electron beams present, a mechanism previously suggested as a possible source of the electron holes. All of these phenomena are detected on closed magnetic field lines, well inside the magnetopause. This was determined by "ion soundings" (Scudder, 2002).

\section{Discussion}

Note that we have not previously addressed the source of the elevated ion and electron backgrounds shown in Figs. 1 and 2. One likely source is pulsed cusp magnetic reconnection (Lee and Fu, 1985; Scholer, 1988) with concomitant ion injections (Lockwood et al., 1998; Boudouridis et al., 2001; Lockwood et al., 2001). In this paper we focus on further heating of electrons of these events.

5.1 The Alfvén wave ponderomotive force and electron heating

The wave ponderomotive force on charged particles has been derived by Landau and Lifschitz (1960), Lee and Parks (1983) and Li and Temerin (1993) and is given below:

$\boldsymbol{F}_{\boldsymbol{P} \boldsymbol{M}}=\frac{q_{s}^{2}}{4 m_{s}} \nabla\left(\frac{1}{\Omega_{s}^{2}-\omega^{2}} E_{\perp}^{2}-\frac{1}{\omega^{2}} E_{\|}^{2}\right)$

where $q_{s}$ is the particle charge, (the subscript "s" is used for species), $m_{s}$ the particle mass, $\Omega_{s}$ the particle gyrofrequency, and $\omega$ is the wave frequency. $\nabla E_{\perp}^{2}$ and $\nabla E_{\|}^{2}$ are the gradients of the wave perpendicular and parallel electric fields. Equation (1) can easily be obtained from the ponderomotive potential $\Phi_{P M}$ (Bhattacharya et al., 1990; Bellan and Stasiewicz, 1998) for a charged particle in a wave field:

$\Phi_{P M}=\left\langle\frac{\boldsymbol{r} \cdot \boldsymbol{E}}{2}\right\rangle=\frac{q_{s}^{2}}{4 m}\left(\frac{1}{\Omega_{s}-\omega^{2}} E_{\perp}^{2}-\frac{1}{\omega^{2}} E_{\|}^{2}\right)$

where $\boldsymbol{r}$ is the position vector of the particle, and the bracket denotes a time average over the wave period. Equation (2) is more suitable to calculate the energy gains for electrons and ions.

In Fig. 4a bottom panel, near the trailing edge of the Alfvén wave, the steepened part is found from 08:25:58 UT to $08: 26: 04 \mathrm{UT}$, or a duration of $\sim 6 \mathrm{~s}$. This corresponds to a frequency of $\sim 0.17 \mathrm{~Hz}$. This is much smaller than the local electron cyclotron frequency $\sim 6.7 \cdot 10^{3} \mathrm{~Hz}$. For obliquely propagating Alfvén waves, we have $E_{\|} \sim E_{\perp} k_{\|} / k_{\perp}$. Thus, the ratio of perpendicular to parallel electric field term in Eq. (1) can be written as:

$\omega^{2} E_{\perp}^{2} / \Omega_{e}^{2} E_{\|}^{2} \approx \omega^{2} k_{\perp}^{2} / \Omega_{e}^{2} k_{\|}^{2}$

so one gets for the ratio of the two terms as: (assuming that $m_{e}^{2} k_{\perp}^{2} / m_{i}^{2} k_{\|}^{2}<1$ and $\left.\omega / \Omega_{i}<1\right)$ :

$\left(m_{e}^{2} k_{\perp}^{2} / m_{i}^{2} k_{\|}^{2}\right)\left(\omega^{2} / \Omega_{i}^{2}\right) \ll 1$

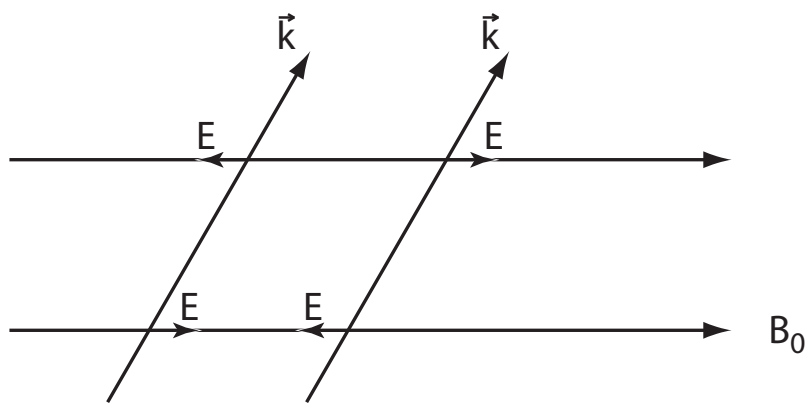

Fig. 11. Two obliquely propagating electromagnetic proton cyclotron waves and their parallel (to $\boldsymbol{B}_{0}$ ) component electric fields. On the top field line, the electric field components are pointing away from each other leading to acceleration of electron beams directed towards each other.

Thus, the electrons should be heated mainly by the gradient of the parallel electric field.

For the event in Fig. 4a with the relevant plasma parameters mentioned in the text, the energy gain can be estimated from Eq. (2). It is seen that the electron can be heated up to $\sim 2 \mathrm{keV}$ in $\sim 6 \mathrm{~s}$, the time period of the steepened edge of the Alfvén wave. This is more than sufficient for the observed energy $(\sim 200 \mathrm{eV})$. These heated electrons are believed to create the magnetic holes through a diamagnetic effect.

We have also calculated the ponderomotive energy gain of ions. For the same wave cycle time $(\sim 6 \mathrm{~s})$, the energy gain is $\sim 1 \mathrm{eV}$. We do not find evidence in the ion data for heating simultaneously with the electrons. This may be due to the more gentle heating process. The existence of proton cyclotron waves is certainly evidence of the possible creation of anisotropic proton heating.

\subsection{Possible sources of Alfvén waves}

One possible source of the Alfvén waves is creation by pulsed magnetic reconnection in the cusp with consequential propagation into the magnetosphere to the spacecraft location. Another possibility is magnetosheath wave penetration into the magnetosphere (however, it should be noted that the frequency spectrum of the boundary layer Alfvén waves was steeper than that for interplanetary Alfvén waves). A third possibility is wave generation via solar wind ram pressure pulses (Haerendel, 1994; Tsurutani et al., 2001b).

\subsection{Bipolar pulse onsets}

The isotropic electron distributions, both in the background and simultaneously with the ion heated events, preclude the possibility of the existence of long-lasting bi-directional electron beams, thought to be a possible free energy source of the bipolar pulse events (Omura, 1996; Kojima et al., 1997; Goldman et al., 1999a, b; Singh et al., 2001; Singh, 2002). 
We would like to point out that there is a possibility to generate bipolar pulses by ion beams. Ashour-Abdallah and Okada (1986) have suggested a mechanism for the generation of broadband electrostatic noise (BEN) in the plasma sheet boundary layer in terms of electron acoustic waves driven by an ion beam. This mechanism requires the presence of two population of electrons (cold and hot) in addition to an ion beam. For the two electron heating events, the hot electrons are evidently present. However, the information about the cold electron component is not available at this time. As shown earlier, a field-aligned ion beam is present during the background interval of the electron heating events, thus there is a distinct possibility of electron acoustic wave excitation by the ion beams. An interesting aspect of the ion beam generation mechanism is that the instability saturates by trapping cold electrons, which eventually become hot, thus shutting off the instability. This mechanism would give rise to phase-space electron holes which will produce bipolar pulse signature.

There is another possible bipolar pulse generation mechanism, which we propose here. The parallel electric field component of the obliquely propagating proton cyclotron waves can provide a mechanism for short duration bi-directional electrons, however. A schematic is shown in Fig. 11.

In the figure, the two proton cyclotron waves are propagating oblique to $\boldsymbol{B}_{0}$. There is a component of the wave electric field parallel to $\boldsymbol{B}_{0}$. The sign of $E$ will oscillate spatially as the wave propagates along the $\boldsymbol{k}$ direction. The two wave packets are independent, and for this case, are shown to have phases $180^{\circ}$ relative to each other. There will be positions (top field line) when the electric fields will accelerate electrons toward each other (creating two oppositely directed beams). At this time, this is only a suggestion. Details of the acceleration and the threshold of a possible cross electron beam instability are currently being investigated.

\section{Proposed scenario}

We have shown many interrelated plasma phenomena in the Polar data set. Our working hypothesis and proposed scenario is the following: magnetic reconnection or interplanetary pressure pulses incident on the magnetopause lead to the generation of large amplitude Alfvén waves which propagate within the boundary layer to the spacecraft. The waves phase-steepened and cause electron heating through the ponderomotive force. The heated electrons cause the magnetic holes through the diamagnetic effect. The Alfvén waves heat protons only slightly. The anisotropic particles generate electromagnetic cyclotron waves through the loss cone instability. The obliquely propagating ion cyclotron waves heat electrons which then generate bipolar pulse events (electron holes).

This paper should be considered as an initial look into a very complex multifaceted physical problem. There are several tens of these electron heating events during this same day, and our next step will be to examine many more of these events to determine if a general, uniform picture of this process emerges.

Acknowledgements. We wish to thank J. D. Scudder and C. T. Russell for the plasma and magnetic field data, respectively. Portions of this research were performed at the Jet Propulsion Laboratory, California Institute of Technology, under contract with National Aeronautics and Space Administration. The research conducted at the University of Iowa was under NASA/GSFC Grants NAG5-7943 and NAG5-9561. BD wishes to acknowledge the NRC/NAS for their support.

\section{References}

Angelopoulos, V., Mozer, F. S., Bonnell, J., Temerin, M., Somoza, M., Peterson, W. K., Collin, H. L., and Giles, B.: Wave power studies of cusp crossings with the Polar satellite, J. Geophys. Res., 106, 5987-6006, 2001.

Ashour-Abdallah, M. and Okuda, H.: Electron-acoustic instabilities in the geomagnetic tail, Geophys. Res. Lett., 13, 366-369, 1986.

Bellan, P. M. and Stasiewicz, K.: Fine scale cavitation of ionospheric plasma caused by inertial Alfvén wave ponderomotive force, Phys. Rev. Lett. 80, 3523-3526, 1998.

Bhattacharya, B., Watanabe, T., and Nishikawa, K.: Single particle and fluid picture for the ponderomotive drift in cold magnetized plasma, J. Phys. Soc. Japan, 59, 2776-2781, 1990.

Boudouridis, A., Spence, H. E., and Onsager, T. G.: Investigation of magnetopause reconnection models using two collocated, lowaltitude satellites: A unifying reconnection geometry, J. Geophys. Res., 106, 29451-29466, 2001.

Cummings, W. D. and Coleman, Jr., P. J.: Magnetic fields in the magnetopause and vicinity at synchronous altitude, J. Geophys. Res., 73, 5699-5718, 1968.

Goldman, M. V., Openheim, M. M., and Newman, D. L.: Nonlinear two-stream instabilities as an explanation for auroral bipolar wave structures, Geophys. Res. Lett., 26, 1821-1824, 1999a.

Goldman, M. V., Openheim, M .M., and Newman, D. L.: Theory of localized bipolar wave-structures and nonthermal particle distributions in the auroral ionosphere, Nonlinear Proc. Geophys., 6, 221-228, 1999b.

Gurnett, D. A. et al.: The Polar plasma wave instrument. Space Sci. Rev. 71, 597-622, 1995.

Haerendel, G.: Acceleration from field-aligned potential drops, Astrophys. J. Suppl. Series, 90, 765-774, 1994.

Kojima, H., Matsumoto, H., Chikuba, S., Horiyama, S., AshourAbdalla, M., and Anderson, R. R.: Geotail waveform observations of broadband/narrowband electrostatic noise in the distant tail, J. Geophys. Res., 102, 14 439-14 455, 1997.

Landau, L. D. and Lifschitz, E. M.: Electrodynamics of Continuous Media, Pergammon Press, Oxford, 64-69, 1960.

Lee, N. C. and Parks, G. K.: Ponderomotive force in a warm twofluid plasma, Phys. Fluids, 26, 724-729, 1983.

Lee, L. C. and Fu, Z. F.: A theory of magnetic flux transfer at the Earth's magnetopause, Geophys. Res. Lett., 12, 105-108, 1985.

Li, X. L. and Temerin, M.: Ponderomotive effects on ion acceleration in the auroral zone, Geophys. Res. Lett., 20, 13-16, 1993.

Lockwood, M., Davis, C. J., Onsager, T. G., and Scudder, J. D.: Modelling signatures of pulsed magnetopause reconnection in cusp ion dispersion signatures seen at middle altitudes, Geophys. Res. Lett., 25, 591-594, 1998. 
Lockwood, M., Milan, S. E., Onsager, T., Perry, C. H., Scudder, J. A., Russell, C. T., and Brittnacher, M.: Cusp ion steps, field aligned currents and polarward moving auroral forms, J. Geophys. Res., 106, 29 555-29 569, 2001.

Lühr, H. and Klöcker, N.: AMPTE-IRM observations of magnetic cavities near the magnetopause, Geophys. Res. Lett., 14, 186189, 1987.

Omura, Y., Matsumoto, H., Miyake, T., and Kojima, H.: Electron beam instabilities as generation mechanisms of electrostatic solitary waves in the magnetotail, J. Geophys. Res., 101, 2685-2697, 1996.

Pickett, J. S., Menietti, J. D., Hospodarsky, G. B., Gurnett, D. A., and Stasiewicz, K.: Analysis of the turbulence observed in the outer cusp boundary layer, J. Atmos. Sol. Terr. Phys., (in press), 2002.

Russell, C. T., Snare, R. C., Means, J. D., Pierce, D., Dearborn, D., Larson, M., Barr, G. and Le, G.: The CGS/POLAR magnetic field investigation, Space Sci. Rev., 71, 563-582, 1995.

Savin, S. P. et al.: Interball tail probe measurements in outer cusp and boundary layers, in Geosp. Mass, Energy Flow: Results Inter. Sol-Terr. Phys. Program, (Eds) Horwitz, J., Gallagher, D., and Peterson, W., AGU, 104, 25-44, 1998.

Scholer, M.: Magnetic flux transfer at the magnetopause based on single X-line bursty reconnection, Geophys. Res. Lett. 15, 291294, 1988.

Scudder, J., Hunsacker, F., Miller, G., Lobell, J., Zawistowski, T., Ogilvie, K., Keller, J., Chornay, D., Herrero, F., Fitzenreiter, R., Fairfield, D., Needall, J., Bodet, D., Googins, J., Kletzing, C., Torbert, R., Vandiver, J., Bentley, R., Fillius, W., McIlwain, C., Whipple, E., and Korth, A.: Hydra - A 3-dimensional electron and ion hot plasma instrument for the Polar spacecraft of the
CGS mission, Space Sci. Rev., 71, 459-495, 1995.

Singh, N., Loo, S. M., Wells, B. E., and Lakhina, G. S.: Evolution of electron beam generated waves resulting in transverse ion heating and filamentation of the plasma, J. Geophys. Res., 106, 21 165-21 181, 2001.

Singh, N.: Space-time evolution of electron-beam driven electron holes and their effects on the plasma, Non. Proc. Geophys., this issue, 2003.

Smith, E. J. and Tsurutani, B. T.: Magnetosheath lion roars, J. Geophys. Res., 81, 2261-2266, 1976.

Sonnerup, B. U. O. and Cahill, L. J.: Magnetopause structure and altitude from Explorer 12 observations, J. Geophys. Res., 72, 171-183, 1967.

Stasiewicz, K., Seyler, C. E., Mozer, F. S., Gustafsson, G., Pickett, J., and Popielawska, B.: Magnetic bubbles and kinetic Alfvén waves in the high-latitude magnetopause boundary, J. Geophys. Res., 106, 29503-29514, 2001.

Sugiura, M., Skillman, T. L., Ledley, B., and Heppner, J. P.: "Holes" in the magnetic field near the magnetopause, EOS Trans, AGU, 50, 278, 1969.

Treumann, R. A., Brostorm, L., LaBelle, J., and Sckopke, N.: The plasma wave signature of a "Magnetic Hole" in the vicinity of the magnetopause, J. Geophys. Res., 95, 19 099-19 114, 1990.

Tsurutani, B. T., Arballo, J. K., Galvan, C., Zhang, L. D., and Zhou, X.-Y.: Polar cap boundary layer waves: An auroral zone phenomena, J. Geophys. Res., 106, 19 035-19 055, 2001 a.

Tsurutani, B. T., Zhou, X.-Y., Vasyliunas, V. M., Haerendel, G., Arballo, J. K., and Lakhina, G.S.: Interplanetary shocks, magnetopause boundary layers and dayside auroras: The importance of a very small magnetospheric region, Surveys in Geophys., 22, 101-130, 2001b. 